\title{
Initial Stroke Thrombectomy Experience in New York City during the COVID-19 Pandemic
}

\author{
(D).A. Yaeger, (D).T. Fifi, (D). Lara-Reyna, (D). Rossitto, (D). Ladner, (D) B. Yim, (D)T. Hardigan, (D) G.A. Maragkos,
} (D) T. Shigematsu, DS. Majidi, and (D). Mocco

\begin{abstract}
SUMMARY: New York City has become the global epicenter of the coronavirus 2019 (COVID-19) pandemic. Despite a massive shift in health care resources, cerebrovascular disease continues to be a substantial burden. We review the first 10 patients undergoing thrombectomy following a series of governmental and institutional policy changes diverting resources to the care of critically ill patients with COVID-19. Ten patients with emergent large-vessel occlusion underwent thrombectomy between March 23 and April 1, 2020. Five patients tested positive for the COVID-19 virus. Successful reperfusion was achieved in 9 of 10 patients, at a median time of 37 minutes from vascular access. The postprocedural NIHSS score improved by an average of 7.7 points. Of the 5 patients positive for COVID-19, none have experienced a critical respiratory illness. We report the early incidence of COVID-19 positivity in patients with emergent large-vessel occlusion and demonstrate that thrombectomy continues to be an efficacious option, as well as safe for health care providers.
\end{abstract}

ABBREVIATIONS: COVID-19 = coronavirus 2019; ELVO = emergent large-vessel occlusion; PPE = personal protective equipment; PUI = patients under investigation for COVID-19

$\mathrm{T}$ he novel coronavirus 2019 (COVID-19) pandemic reached global magnitude within 3 months, affecting billions of people worldwide and compromising the most modern health care systems. Despite recognition by the World Health Organization as a public health emergency on January $30,2020,{ }^{1}$ the virus spread internationally, with the first known case of COVID-19 in New York City occurring on March 1, 2020. Since then, as of this writing on April 5, 2020, there have been 67,551 cases and 2256 deaths in New York City, which has rapidly become the global epicenter of this devastating pandemic. ${ }^{2}$ Many early reports from China and Italy have suggested a high incidence of neurologic pathology among patients with COVID-19. ${ }^{3-6}$ The virus is associated with a hyperinflammatory and hypercoagulable state; ${ }^{7,8}$ thus, the incidence of stroke among patients with the novel coronavirus is hypothesized to be higher than normal. ${ }^{9}$ Two retrospective studies from China observed ischemic strokes complicating $>5 \%$ of COVID-19 infections. ${ }^{10,11}$

Received April 22, 2020; accepted after revision May 18.

From the Department of Neurosurgery, Mount Sinai Health System, New York, New York.

Please address correspondence to Kurt A. Yaeger, MD, 1 Gustav Levy Pl, Annenberg Building 8th Floor, New York, NY; e-mail: Kurt.yaeger@mountsinai.org; @Dr_Yaeger

- Indicates open access to non-subscribers at www.ajnr.org

http://dx.doi.org/10.3174/ajnr.A6652
The goals of this report are the following: 1) document the prevalence of COVID-19 in patients presenting with emergent large-vessel occlusion (ELVO) during the early COVID-19 period; 2) determine whether initial outcomes of such patients undergoing thrombectomy are consistent with prior experience; 3) determine whether performing thrombectomy would result in COVID-19 infection of involved health care workers; and 4) discuss changes to the overall stroke workflow in adapting to the high infectious concerns and the diversion of health care resources.

\section{MATERIALS AND METHODS}

We reviewed the medical records of patients who underwent stroke thrombectomy for ELVO during a 9-day time span from March 23 to April 1, 2020, within our multi-institutional health care system. This analysis was cleared by the institutional review board, and written patient consent was waived.

Throughout the study period, the triage of patients with concern for ELVO was similar to that in the prepandemic protocol. ${ }^{12}$ During the new COVID stroke triage process (Fig 1), the consulting stroke team would identify risk factors for COVID-19 infection, including clinical or social indicators such as fever, cough, or symptomatic personal contacts. Patients for intervention were triaged as either patients under investigation for COVID-19 (PUI) or non-PUI. Angiography suites were prepared with full airborne precautions. All endovascular devices and anesthesia 


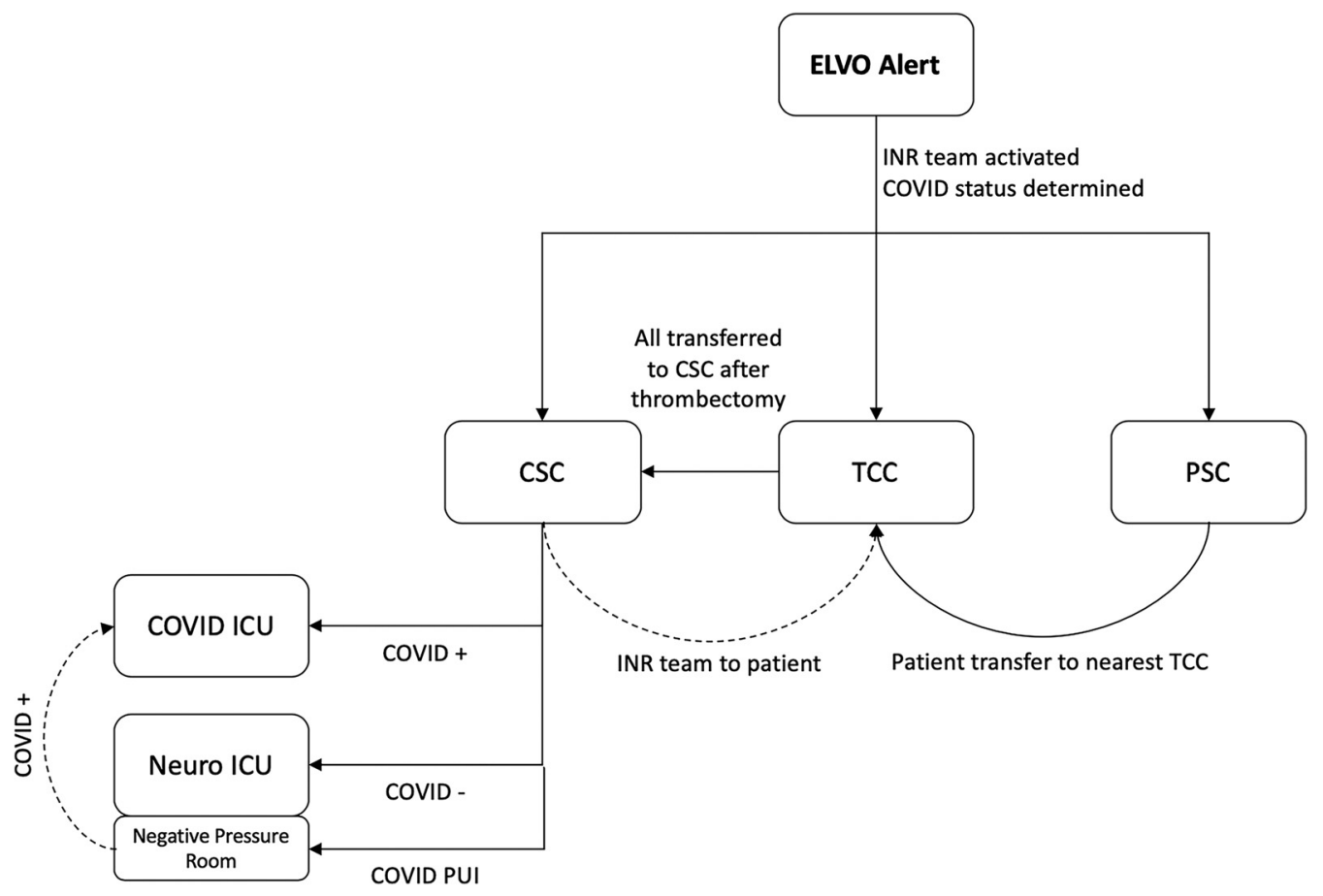

FIG 1. Our health system workflow triaging patient transfer for thrombectomy. First, COVID-19 status is interrogated concurrently with the initial work-up. If the patient presents to a primary stroke center, he or she is transferred to the nearest thrombectomy-capable center. Concurrently, if necessary, the endovascular team mobilizes to the target center. Postprocedurally, all patients are transferred to the centralized comprehensive stroke center. If confirmed positive for COVID-19 infection, the patient is transferred to the dedicated COVID intensive care unit for respiratory and neurologic monitoring. If negative, the patient can recover in the standard neurosurgical intensive care unit. PUI whose test results are pending, immediately after thrombectomy, are placed in negative pressure rooms in the neurosurgical intensive care unit and transferred to the COVID-19 intensive care unit if the results return positive. ICU indicates intensive care unit; INR, interventional neuroradiology; CSC, comprehensive stroke center; TCC, thrombectomy-capable center; PSC, primary stroke center.

supplies not needed for the procedure were removed. The minimum number of health care providers was used, and all active personnel donned full airborne personal protective equipment (PPE: N95 mask, face shield, gown, and gloves). PPE was donned at the beginning of each case before the patient entered the angiography suite. In general, local anesthesia or mild sedation was preferred for each patient, with conversion to general anesthesia performed only for patients with an inability to protect the airway.

All patients regardless of respiratory symptoms have had surgical masks in place for droplet precautions, and patients who were aphasic or neurocognitively compromised were treated as PUI. To minimize aerosolization in the angiography suite and subsequent exposure to the team and equipment, anesthesiology teams requested that patients who may require general anesthesia be intubated by a rapid response team (for inpatients) or emergency department staff and brought directly to the interventional suite either with bag-valve-mask with a viral filter or a portable ventilator. If intubation was required during the procedure, it was performed by the attending anesthesiologist, with all other members of the team outside the room for the duration of this aerosolizing procedure. At the time of skin preparation and draping, the proceduralist and assistant donned sterile gowns and surgical gloves and maintained full airborne precautions from the neck up. Thrombectomy procedures were performed in routine fashion using standard devices, including aspiration catheters and stent retrievers. Any endovascular devices or anesthesia equipment that was not immediately accessible within the room was acquired by a runner outside the room and handed off to the procedure team, preventing the need for repeat donning and doffing of PPE. Following thrombectomy, all patients were transferred to a single comprehensive stroke center.

\section{RESULTS}

\section{Patient Characteristics}

Between March 23 and April 1, 2020, confirmed cases of COVID-19 in New York City rose by $301 \%$, from 20,875 to 83,712 , with reported deaths caused by the disease increasing from 157 to 1941 (1136\%). ${ }^{13}$ During this time, 10 patients underwent endovascular thrombectomy in our health care system (Table). The average NIHSS score on presentation was 14 (range, 10-19). A variety of intracranial large-vessel occlusions were observed. The average time from last known well to stroke code was 5.5 hours (range, 30 minutes to 20 hours). Five patients received intravenous thrombolysis with $\mathrm{tPA}$.

Preoperatively, only 1 patient tested positive for COVID-19. Although no patients had fever on admission, 3 patients had clinical symptoms including cough and dyspnea. Only 1 patient had known contact with persons positive for COVID-19. Based on either clinical or radiographic concerns for infection, 9 patients 


\begin{tabular}{|c|c|}
\hline Characteristics & \\
\hline \multicolumn{2}{|l|}{ Patients } \\
\hline Male sex & $6(60 \%)$ \\
\hline Age (mean) (range) (yr) & $64(37-90)$ \\
\hline Presenting NIHSS score (mean) (range) & $14(10-19)$ \\
\hline \multicolumn{2}{|l|}{ Presenting hospital borough } \\
\hline Queens & 6 \\
\hline Manhattan & 3 \\
\hline Brooklyn & 1 \\
\hline \multicolumn{2}{|l|}{ Occlusion location } \\
\hline Right ICA & 1 \\
\hline Left ICA & 1 \\
\hline Right M1 & 3 \\
\hline Left M1 & 2 \\
\hline Right $M 2$ & 1 \\
\hline Left M2 & 1 \\
\hline Basilar artery & 1 \\
\hline Intravenous thrombolysis (tPA) & 5 \\
\hline \multicolumn{2}{|l|}{ COVID-19 features } \\
\hline Symptoms & 3 \\
\hline Radiographic & 2 \\
\hline Known sick contact & 1 \\
\hline \multicolumn{2}{|l|}{ COVID-19 PCR test } \\
\hline Positive preprocedure & 1 \\
\hline Positive postprocedure & 4 \\
\hline Negative & 4 \\
\hline Not tested & 1 \\
\hline
\end{tabular}

Note:-PCR indicates polymerase chain reaction.

were tested for COVID-19 via nasopharyngeal swab, and 5 ultimately had a positive test ( $50 \%$ of total cohort).

\section{Preprocedural Workflow}

Seven patients presented initially to a thrombectomy-capable center, whereas 3 presented to a primary stroke center requiring transfer for intervention. Of the 5 patients positive for COVID19, 3 presented to hospitals in Queens, 1 in Brooklyn, and 1 in Manhattan, reflecting an increased COVID-19 prevalence in Queens (33.4\% of cases citywide as of April 3, 2020). ${ }^{14}$

Two patients ( 1 confirmed positive for COVID-19, 1 patient under investigation based on symptoms) were intubated before thrombectomy; the remaining patients underwent minimal sedation. At this writing, none of the interventional team (including technologists, nurses, or clinicians) have experienced symptoms of COVID-19 infection.

\section{Procedural Details}

The median time from endovascular consultation to vascular access was 65 minutes (range, 21-363 minutes). One patient presented with an occluded flow-diverting stent placed 7 days prior for an unruptured aneurysm; the occlusion could not be traversed with a guidewire or catheter, and reperfusion was not achieved. In the 9 other patients, successful reperfusion (defined as TICI $\geq 2 \mathrm{~b}$ ) was achieved with median time of 37 minutes (range, 1470 minutes) from vascular access. Overall, the average time from last known well to reperfusion was 7.2 hours (range, 2-19 hours) in the 9 patients with successful reperfusion. On postoperative day 1 , the NIHSS score was reduced by an average of 7.7 points (median, 8.5 ; range, $0-18$ points).

\section{DISCUSSION}

The ongoing COVID-19 pandemic is a global black swan event, unpredicted but highly consequential, with implications beyond current expectations. As cases surge worldwide, health care institutions have been overrun with patients, some of whom are critically ill on presentation. In response, systems of care have been dramatically altered to accommodate a constant deluge of patients with acute respiratory failure. ${ }^{9}$ Nevertheless, the burden of cerebrovascular disease continues: During a 9-day period, we performed 10 thrombectomy procedures on patients with emergent large-vessel occlusion, 5 of whom ultimately tested positive for COVID-19. The procedural results during this time period are consistent with our pre-COVID institutional experience. These results have been achieved with careful preparation of the angiography suite for incoming patients, safeguards with appropriate $\mathrm{PPE}$, and reorganization of our institutional network to provide thrombectomy at satellite centers and subsequently centralize postoperative care. It is critical to note that no members of the procedural teams have developed symptoms consistent with COVID-19; however, given the lack of long-term follow-up in this report as well as the long incubation time for COVID-19, providers may eventually develop infectious symptoms. Even despite a lack of symptoms, members of the comprehensive stroke team may have been asymptomatic carriers; therefore, the importance of PPE extends to preventing infection among the team.

Rapid changes in citywide health care system processes at the onset of the New York City pandemic led to necessary adaptations to our acute stroke triage process. Early reports on the hypercoagulable effects of the COVID-19 syndrome have been corroborated by our findings of increased incidences of large-vessel occlusion in the early COVID-19 period (data accepted for publication). We have also observed ELVO as the presenting symptom of COVID-19 in several young patients. ${ }^{15}$ As a result of these demographic shifts, we have had a higher index of suspicion for ELVO in a wider array of patients presenting with neurologic symptoms. As the COVID-19 incidence began to exponentially increase across the city, several institutions began to divert patients without COVID-19 with acute stroke to our center, a disruption in the normal referral pattern that has led to a delay in care for several patients with stroke. Thus, we suggest a regional consolidation of thrombectomy care to a smaller number of institutions, thereby preventing time delays for satellite hospitals in finding an accepting center with capacity.

Post-thrombectomy care has changed substantially since the study period began. With neurointensive care faculty reassigned to a COVID-19-focused intensive care unit, an endovascular team member has assumed the role of neurointensivist. This has been feasible only by centralizing the post-thrombectomy care to the comprehensive stroke center, differing from our traditional system, in which patients who undergo acute stroke interventions at a thrombectomy-capable hospital are admitted to the local intensive care unit and are cared for by the in-house stroke team.

Our focus throughout has been the continued delivery of timely stroke care, with great attention to the safety of health care personnel. We have compensated for the diversion of hospital resources toward critically ill patients with COVID-19 by transferring post-thrombectomy patients out of resource-critical 
environments. New York City has become the global epicenter of the COVID-19 pandemic, preceding other US cities by weeks to months. While we present our findings at the early stages of this public health crisis, changes in stroke demographics, incidence, and outcomes may occur as the COVID-19 outbreak continues in various stages of development. Therefore, more longitudinal studies should be undertaken as time passes. However, with this early report, we hope to encourage the continued care of patients with acute stroke with ELVO because we have observed thrombectomy being performed in a safe, effective manner during this crisis. In light of the recently published guidelines on COVID-19 and acute stroke care from the American Heart Association/American Stroke Association, ${ }^{16}$ Society of NeuroInterventional Surgery, ${ }^{17}$ and Society of Vascular and Interventional Neurology, ${ }^{18}$ we report our early clinical experience to corroborate these recommendations and advocate for their strict adherence, to continue improving patient outcomes and health care worker well-being.

Disclosures: Johanna Fif_-UNRELATED: Consultancy: Stryker, Penumbra; Grants/ Grants Pending: Stryker, Penumbra, Microvention. * Money paid to institution.

\section{REFERENCES}

1. Emergency Committee Regarding the Outbreak of Novel Coronavirus (2019-nCoV). Statement on the second meeting of the International Health Regulations. 2005. https://www.who.int/news-room/detail/2301-2020-statement-on-the-meeting-of-the-international-healthregulations-(2005)-emergency-committee-regarding-the-outbreakof-novel-coronavirus-(2019-ncov). Accessed April 5, 2020

2. Johns Hopkins Coronavirus Resource Center. COVID-19 Dashboard. https://coronavirus.jhu.edu/map.html. Accessed April 5, 2020

3. Madjid M, Safavi-Naeini P, Solomon SD, et al. Potential effects of coronaviruses on the cardiovascular system: a review. JAMA Cardiol 2020; Mar 27. [Epub ahead of print] CrossRef Medline

4. Sun T, Guan J. Novel coronavirus and central nervous system. Eur J Neurol 2020 Mar 26. [Epub ahead of print] CrossRef Medline

5. Tan YT, Wang JW, Zhao K. Preliminary recommendations for surgical practice of neurosurgery department in the central epidemic area of 2019 coronavirus infection. Curr Med Sci 2020;40:281-84 CrossRef Medline
6. Zoia C, Bongetta $\mathrm{D}$, Veiceschi $\mathrm{P}$, et al. Neurosurgery during the COVID-19 pandemic: update from Lombardy, Northern Italy. Acta Neurochir (Wien) 2020;162:1221-22 CrossRef Medline

7. Wang D, Hu B, Hu C, et al. Clinical characteristics of 138 hospitalized patients with 2019 novel coronavirus-infected pneumonia in Wuhan, China. JAMA 2020;323:106 CrossRef Medline

8. Guo W, Li M, Dong Y, et al. Diabetes is a risk factor for the progression and prognosis of COVID-19. Diabetes Metab Res Rev 2020 Mar 31. [Epub ahead of print] CrossRef Medline

9. AHA/ASA Stroke Council Leadership. Temporary emergency guidance to US stroke centers during the COVID-19 pandemic. Stroke 2020;51:1910-12 CrossRef Medline

10. Mao L, Wang M, Chen S, et al. Neurological manifestations of hospitalized patients with COVID-19 in Wuhan, China: a retrospective case series study. JAMA Neurol 2020 Apr 10. [Epub ahead of print] CrossRef Medline

11. Li Y, Wang M, Zhou Y, et al. Acute cerebrovascular disease following COVID-19: a single center, retrospective, observational study. January 2020. https://www.researchgate.net/publication/340154622 Acute_Cerebrovascular_Disease_Following_COVID-19_A_Single_ Center_Retrospective_Observational_Study. Accessed April 5, 2020

12. Wei D, Oxley TJ, Nistal DA, et al. Mobile interventional stroke teams lead to faster treatment times for thrombectomy in large vessel occlusion. Stroke 2017;48:3295-3300 CrossRef Medline

13. New York State Department of Health. County by County Breakdown of Positive Cases. https://coronavirus.health.ny.gov/countycounty-breakdown-positive-cases. Accessed April 5, 2020

14. NYC Health. COVID-19. https://www1.nyc.gov/site/doh/covid/covid19-data.page. Accessed April 5, 2020

15. Oxley TJ, Mocco J, Majidi S, et al. Large-vessel stroke as a presenting feature of COVID-19 in the young. N Engl J Med 2020;382:e60 CrossRef Medline

16. On Behalf of the AHA/ASA Stroke Council Leadership. Temporary Emergency Guidance to US Stroke 18 Centers During the COVID19 Pandemic. April 2020. https://doi.org/10.1161/STROKEAHA.120.030023. Accessed April 5, 2020

17. Fraser JF, Arthur AS, Chen M, et al. Society of NeuroInterventional Surgery recommendations for the care of emergent neurointerventional patients in the setting of Covid-19. J Neurointerv Surg 2020;12:539-41 CrossRef Medline

18. Nguyen TN, Abdalkader M, Jovin TG, et al. Mechanical thrombectomy in the era of the COVID-19 pandemic: emergency preparedness for neuroscience teams: a guidance statement from the Society of Vascular and Interventional Neurology. Stroke 2020;51: 1896-1901 CrossRef Medline 